\title{
Maternal dietary patterns in pregnancy and the association with small-for-gestational-age infants
}

\author{
John M. D. Thompson ${ }^{1}$, Clare Wall ${ }^{2}$, David M. O. Becroft ${ }^{1}$, Elizabeth Robinson ${ }^{3}$, Chris J. Wild ${ }^{4}$ \\ and Edwin A. Mitchell ${ }^{1}$ \\ ${ }^{1}$ Department of Paediatrics, University of Auckland, Private Bag 92019 Auckland, New Zealand \\ ${ }^{2}$ Department of Nutrition, University of Auckland, Auckland, New Zealand \\ ${ }^{3}$ Department of Epidemiology and Biostatistics, Faculty of Medical and Health Sciences, University of Auckland, Auckland, \\ New Zealand \\ ${ }^{4}$ Department of Statistics, Faculty of Science, University of Auckland, Auckland, New Zealand
}

(Received 17 December 2008 - Revised 26 November 2009 - Accepted 27 November 2009 - First published online 9 March 2010)

\begin{abstract}
Maternal nutritional status before and during pregnancy is important for the growth and development of the fetus. The effects of pre-pregnancy nutrition (estimated by maternal size) are well documented. There is little information in today's Western society on the effect of maternal nutrition during pregnancy on the fetus. The aim of the study was to describe dietary patterns of a cohort of mothers during pregnancy (using principal components analysis with a varimax rotation) and assess the effect of these dietary patterns on the risk of delivering a small-for-gestationalage (SGA) baby. The study was a case-control study investigating factors related to SGA. The population was 1714 subjects in Auckland, New Zealand, born between October 1995 and November 1997, about half of whom were born SGA ( $\leq 10$ th percentile for sex and gestation). Maternal dietary information was collected using FFQ after delivery for the first and last months of pregnancy. Three dietary patterns (traditional, junk and fusion) were defined. Factors associated with these dietary patterns when examined in multivariable analyses included marital status, maternal weight, maternal age and ethnicity. In multivariable analysis, mothers who had higher 'traditional' diet scores in early pregnancy were less likely to deliver a SGA infant $(\mathrm{OR}=0.86$; $95 \%$ CI $0.75,0.99)$. Maternal diet, particularly in early pregnancy, is important for the development of the fetus. Socio-demographic factors tend to be significantly related to dietary patterns, suggesting that extra resources may be necessary for disadvantaged mothers to ensure good nutrition in pregnancy.
\end{abstract}

Maternal nutrition: Nutritional epidemiology: Small for gestational age: Dietary patterns: Pregnancy

Adequate nutritional status before pregnancy is essential for optimal development and growth of the fetus. In terms of pre-pregnancy nutritional status, most commonly measured by maternal stature, it has been shown that maternal size (weight) before pregnancy has one of the most significant effects on birth weight ${ }^{(1)}$. Data from the Auckland Birthweight Collaborative study have previously confirmed such a relationship in the population studied here ${ }^{(2)}$.

Also of notable importance is nutritional intake during pregnancy, for which there is relatively little literature particularly in human subjects. Most information in this field comes from trials of supplementation in developing countries rather than in 'Western' society where nutrition is considered more adequate or from animal studies where manipulation of the diet can be undertaken and the effect on birth outcome studied.

Data from the Dutch famine of 1944-5 during the war ${ }^{(3-6)}$ demonstrated that exposure to famine during the third trimester, the time of greatest growth of the fetus, reduced birth weight by about $10 \%$. Exposure to famine in early or mid pregnancy did not appear to have an effect on birth weight ${ }^{(4,7)}$. However, such studies are relatively extreme and these environments are rare in Western society today. Most work has been carried out in animals with restriction of diet in early and late pregnancy showing large effects in terms of outcomes at birth ${ }^{(8-11)}$ and development of disease in later life. However, how these animal data translate to nutrition during pregnancy in human subjects still remains unclear.

Current recommendations for nutritional requirements for pregnancy are limited, as they are based on supplementary intake in addition to the estimated requirements for nonpregnant women. It is recognised that the nutritional health of the mother before pregnancy may be as important as her nutrition throughout pregnancy. An important example of this is dietary folate, a lack of which is recognised as leading to increased risk of developing neural tube defects ${ }^{(12-15)}$.

The effects of maternal diet in pregnancy and disease of the resulting offspring in adult life would appear to be dependent on the combination of maternal diet and the dietary exposure of the infant child and adult ${ }^{(16,17)}$. 
Identification of dietary patterns has become a useful tool in epidemiological studies that seek to explore the relationship between dietary exposures and health outcome. The use of an FFQ in the present study to determine dietary habits enables a longer term view of dietary intakes as opposed to using a $24 \mathrm{~h}$ dietary recall or food diary method. This approach to dietary analyses is population specific; thus, making it suitable for use in cohort studies that aim to describe relationships between dietary exposures and health outcomes over periods of time. It also has the advantage of reducing a large number of individual variables to a more manageable number of summary variables. An analysis of specific nutrients or foods to risk of disease is limited because of the inter-correlations between nutrients and/or food groups. Dietary patterns as defined by principal components analysis avoid this problem and describe overall diet more generally and can also be used to estimate the risk of disease and have the advantage of being amenable to the formation of subsequent public health recommendations.

The primary aim of the present study was to identify risk factors for small-for-gestational-age (SGA), in term infants, particularly those factors that may be modifiable ${ }^{(2)}$. A previous analysis from the present study relating food groups in the maternal diet identified fish, carbohydrate and folate supplementation in early pregnancy as reducing the risk of $\mathrm{SGA}^{(18)}$.

This report analyses diet using food items from FFQ to identify dietary patterns. It assesses the relationship of dietary patterns with socio-demographic and obstetric factors and the effect of dietary patterns on having an SGA infant.

\section{Methods}

The Auckland Birthweight Collaborative study was designed to identify risk factors for SGA infants. The methodology has been described in detail previously ${ }^{(2)}$. In brief, it was a case-control study carried out in the Auckland and Waitemata District Health Boards in Auckland, New Zealand. Babies born at term (completed gestation of 37 or more weeks) between 16 October 1995 and 12 August 1996 from both regions were eligible and between 13 August 1996 and 30 November 1997 in the Auckland District Health Board only. All infants born SGA as defined by national population birth weight percentile ${ }^{(19)}(\leq 10$ th percentile) were selected and a random sample of appropriate-for-gestational-age infants were selected as controls.

Information was collected by (1) a maternal interview that collected data on socio-demographic variables, obstetric history and detailed information about the index pregnancy, (2) data extracted from the electronic obstetric notes, (3) macroscopic and microscopic examination of the placenta and (4) two maternally self-completed FFQ.

The FFQ were completed shortly after the birth of the infant. They were semi-quantitative FFQ, identical and derived from the Life in New Zealand study questionnaires ${ }^{(20)}$. One FFQ asked the mothers about their frequency of consumption of individual foods in the first month of pregnancy and the other questionnaire about the last month before delivery of their baby. Questions were asked about individual foods in the broad categories: fresh fruits; vegetables; rice/noodles; chicken, meat or substitutes; dairy foods; puddings; cereals, cakes and biscuits; spreads, sauces and garnishes; snacks and takeaways; drinks. The questions on each food were asked in the following manner 'At about the time you became pregnant how often did you eat a serving of...' for the first month of pregnancy and 'During the last 4 weeks how often did you have a serving of...' for the last month of pregnancy. The choices were (1) not at all, (2) less than once a week, (3) once a week, (4) a few times a week, (5) once a day and (6) a few times a day. The questionnaire collected information on seventy-one food items.

\section{Statistical methods}

The analysis to determine characteristics of dietary patterns was carried out using all seventy-one food items from the early pregnancy questionnaire. This analysis was carried out using a principal components analysis with a varimax rotation using PROC FACTOR in Statistical Analysis Systems version 9.1 (SAS Institute, Cary, NC, USA). Using a varimax rotation ensured that the distributions of the scores would be centred around 0 , with a standard deviation of 1 . This allows easy interpretation of the results, as the point estimates from regression analyses will be equivalent to a change of the magnitude of the equivalent number of standard deviations.

A scree plot was used to determine the number of factors, along with the percentage of variance explained by each factor. The distribution of the loadings across dietary patterns was examined to ensure that there was no great overlap between factors. Factor loadings were considered to have a strong association when these had a magnitude of 0.3 or greater, and the foods within a factor with loadings of these values are considered to be descriptive of the 'pattern' of diet associated with this factor.

The weightings were then used to create scores for each woman for each of the three diets for both early and late pregnancy. In order to ensure the same interpretability of the results, we standardised the late pregnancy distributions so that the dietary scores would also have a mean of 0 and standard deviation of 1 . Using the same weightings for early and late pregnancies enabled us first to have consistent dietary patterns across the pregnancy and second to assess the correlation between the time points to assess the change in diet from early to late pregnancy.

Associations between pattern of diet and independent predictors were assessed by linear regression. Because of the disproportionate sampling of the SGA infants at birth, analyses were weighted using PROC SURVEYREG in Statistical Analysis Systems (SAS Institute). Variables associated with each diet at the $10 \%$ level in univariable analyses were included in a multivariable analysis.

To determine associations between dietary pattern and SGA, logistic regression was carried out with the individual diet factor scores used as independent variables. OR describe the change in risk of an SGA infant associated with a unit change (1 SD) in the diet score. Finally, the significant dietary scores were added to our previously published multivariable model of risk factors for SGA to assess whether dietary score had an additional effect to those variables already controlled for. This analysis controlled for gestational age, infant sex, maternal smoking during pregnancy, maternal height and pre-pregnancy weight, primiparity, ethnicity and maternal hypertension. 
Table 1. Loading factors from factor analysis using varimax rotation for early pregnancy (a negative factor means the diet was less likely to have this food)

\begin{tabular}{|c|c|c|c|}
\hline \multirow[b]{2}{*}{ Food } & \multicolumn{3}{|c|}{ Early pregnancy } \\
\hline & Fusion & Junk & Traditional \\
\hline $\begin{array}{l}\text { Percentage of } \\
\text { variation explained }\end{array}$ & $4.96 \%$ & $4.52 \%$ & $4.36 \%$ \\
\hline Avocados & 0.05989 & -0.00338 & $0.35023^{*}$ \\
\hline Berry fruits & $0 \cdot 20190$ & 0.10609 & 0.34340 * \\
\hline Stone fruits & 0.25774 & 0.05226 & $0.35746^{\star}$ \\
\hline Apples/pears & $0.39593^{*}$ & 0.01690 & $0.47179^{*}$ \\
\hline Citrus fruits & $0.34872^{*}$ & -0.02648 & $0.44923^{*}$ \\
\hline Kiwi/feijoa & $0.33781^{*}$ & 0.02160 & $0.33764^{*}$ \\
\hline Melon & $0.35940^{*}$ & 0.08943 & 0.21695 \\
\hline Bananas & $0.34450^{*}$ & 0.11129 & $0.41833^{\star}$ \\
\hline Dried fruits & 0.01396 & -0.07569 & $0.42527^{*}$ \\
\hline Tinned fruit & $0.39723^{*}$ & 0.20196 & 0.19130 \\
\hline Tinned in water & $0.35111^{*}$ & 0.08013 & 0.16012 \\
\hline Fruit pies & $0.32507^{*}$ & 0.26801 & 0.14280 \\
\hline Green vegetables & 0.08925 & -0.19655 & $0.53287^{*}$ \\
\hline Root vegetables & 0.04716 & -0.01334 & $0.58861^{*}$ \\
\hline Peas/maize/lentils & 0.18251 & 0.06739 & $0.40458^{\star}$ \\
\hline Potato etc. & 0.04236 & 0.24823 & 0.35930 * \\
\hline Fried rice/noodles & $0.49956^{*}$ & 0.14764 & -0.05139 \\
\hline Boiled rice/pasta & $0.48352^{*}$ & -0.20052 & 0.02018 \\
\hline Lean meat & 0.23086 & -0.01157 & 0.21724 \\
\hline Chicken with skin & $0.36294^{*}$ & 0.07390 & -0.07340 \\
\hline Meat with fat & 0.19558 & 0.23684 & -0.06090 \\
\hline Fish/shellfish & $0.56350^{*}$ & -0.02158 & 0.06023 \\
\hline Made-up meat dishes & $0.39759^{*}$ & 0.12822 & 0.01838 \\
\hline Small goods & 0.25106 & $0 \cdot 27122$ & 0.05043 \\
\hline Vegetarian substitute $†$ & 0.02997 & 0.08514 & $0.35895^{\star}$ \\
\hline Soup with meat & $0.66914^{*}$ & 0.12545 & 0.00295 \\
\hline Cream cheese & 0.00072 & 0.00238 & 0.18977 \\
\hline Hard cheeses & $-0.30063^{*}$ & 0.08763 & $0.39377^{*}$ \\
\hline Soft cheeses & -0.07805 & 0.01428 & 0.21978 \\
\hline Reduced fat cheese & -0.21617 & -0.00753 & $0.39117^{*}$ \\
\hline Cream & 0.06409 & $0 \cdot 20721$ & 0.15999 \\
\hline Condensed milk & $0.35642^{*}$ & 0.04433 & 0.07087 \\
\hline Dairy food/yogurt & 0.14356 & 0.11216 & $0.35464^{*}$ \\
\hline Milk pudding & 0.27775 & 0.03523 & 0.05708 \\
\hline Fruit pudding & 0.16049 & 0.13666 & 0.17698 \\
\hline Cake-type pudding & 0.22111 & 0.28100 & 0.09154 \\
\hline Jelly & $0.40233^{*}$ & 0.21676 & -0.00817 \\
\hline Ice cream & $0.36322^{*}$ & $0.45168^{*}$ & 0.11809 \\
\hline Plain biscuits & 0.01820 & $0.33225^{*}$ & $0.32608^{*}$ \\
\hline Sweet biscuits & 0.01108 & $0.52291^{*}$ & 0.14949 \\
\hline Scones & 0.12196 & $0.39281^{*}$ & 0.19444 \\
\hline Croissants & 0.19561 & 0.16656 & 0.15080 \\
\hline Cakes & 0.29754 & $0.46053^{*}$ & 0.04529 \\
\hline Bread & -0.22284 & 0.27757 & 0.35569 * \\
\hline Porridge & $0.32426^{*}$ & 0.12124 & 0.12738 \\
\hline Cereal & -0.04021 & 0.22766 & 0.23721 \\
\hline Sweetened cereal & 0.21090 & $0.33057^{*}$ & -0.02578 \\
\hline Sweet spreads & -0.19583 & $0.30498^{*}$ & 0.29615 \\
\hline Sauces & -0.14933 & $0.39517^{*}$ & $0 \cdot 24862$ \\
\hline Salad dressing & -0.14341 & 0.28411 & $0.32649^{*}$ \\
\hline Savoury spreads & -0.18416 & $0 \cdot 23880$ & $0 \cdot 21248$ \\
\hline Crisps & -0.00907 & $0.46637^{*}$ & 0.05501 \\
\hline Instants noodles & $0.34801^{*}$ & 0.29805 & -0.00487 \\
\hline Pies & 0.15281 & $0.49800^{*}$ & -0.12437 \\
\hline Crackers & 0.04217 & 0.27793 & 0.26222 \\
\hline Muesli bar & -0.02712 & $0 \cdot 21643$ & 0.22880 \\
\hline Chocolate bar & 0.00852 & $0.66536^{*}$ & -0.08149 \\
\hline Lollies & 0.04877 & $0.63642^{*}$ & -0.08341 \\
\hline Roll up $\ddagger$ & 0.16711 & 0.23064 & -0.01586 \\
\hline Ice blocks & 0.18164 & $0.55762^{*}$ & -0.07295 \\
\hline Butter/margerine $v$. none & -0.02444 & -0.22033 & 0.18842 \\
\hline Eggs & 0.10479 & 0.04371 & 0.04799 \\
\hline Milk & $0.42002^{*}$ & 0.06866 & 0.13417 \\
\hline Fruit juice/cordial & 0.04509 & $0 \cdot 22985$ & 0.24688 \\
\hline
\end{tabular}

Table 1. Continued

\begin{tabular}{lccc}
\hline & \multicolumn{3}{c}{ Early pregnancy } \\
\cline { 2 - 4 } Food & Fusion & Junk & Traditional \\
\hline Water & 0.08344 & -0.18521 & $0.37147^{\star}$ \\
Milo§ & $0.30575^{\star}$ & $0.34992^{\star}$ & 0.03566 \\
Coffee/tea & $-0.44878^{*}$ & 0.12730 & 0.20460 \\
Decaffeinated beverages & 0.01030 & 0.04517 & 0.13391 \\
Sherry/wine & $-0.33950^{\star}$ & -0.02927 & 0.23642 \\
Beer & -0.15422 & 0.11858 & 0.08722 \\
Spirits & -0.19884 & 0.03141 & 0.10432 \\
\hline
\end{tabular}

* Loading factors above 0.3 .

† Vegetarian substitute for meat such as tofu.

$\ddagger$ Roll up - extruded fruit snack.

$\S$ Milo - malted chocolate milk drink.

All analyses were carried out using Statistical Analysis Systems version 9.1 for windows (SAS Institute).

\section{Subjects}

Interview data were available for 1714 subjects of which 844 had been born SGA and 870 appropriate for gestational age. Nutritional questionnaires were returned for 1209 (71\%) women and $723(83 \%)$ European women. There were seventy-five early pregnancy questionnaires and seventy-three late pregnancy questionnaires that were incomplete and could not be analysed, leaving 1134 questionnaires for early pregnancy and 1136 questionnaires for late pregnancy diets available for analysis.

\section{Results}

\section{Total sample}

Analysis of data from early pregnancy indicated that three dietary patterns best described the dietary pattern of the women in the present study. For descriptive purposes, we have named the dietary patterns 'junk', 'traditional' and 'fusion'. The list of foods and the loading factors are shown in Table 1. Of the seventy-one foods included in the analyses, fifty had a loading of greater than 0.3 for at least one of the three dietary patterns. For the 'junk' diet, foods identified as being characteristic included ice cream, sweet biscuits, scones, cakes, sweetened cereal, crisps, pies, lollies, chocolate bars, ice blocks and milo (chocolate energy drink). A traditional New Zealand diet is similar to a 'traditional' British diet, which is meat (lamb in particular), potatoes, carrots (and other root vegetables), peas, gravy, and meat dishes such as cottage pie. The 'traditional' diet included apples/pears, citrus fruit, kiwifruit/feijoas, bananas, green vegetables, root vegetables, peas/maize, dairy food/yogurt and water. The 'fusion' diet is one that combines elements of Asian cuisine with the addition of Western foods. The 'fusion' diet included fruits, fried rice/ noodles, boiled rice/pasta, fish/shellfish, milk and negative loading for tea/coffee, sherry/wine and hard cheeses.

The distributions of the diet scores are shown in Fig. 1.

An analysis of the late pregnancy data showed similar dietary patterns (data not shown). Due to this reason and to avoid confusion of similar diets at two time points, we chose to apply the weightings from the early pregnancy diets to the 


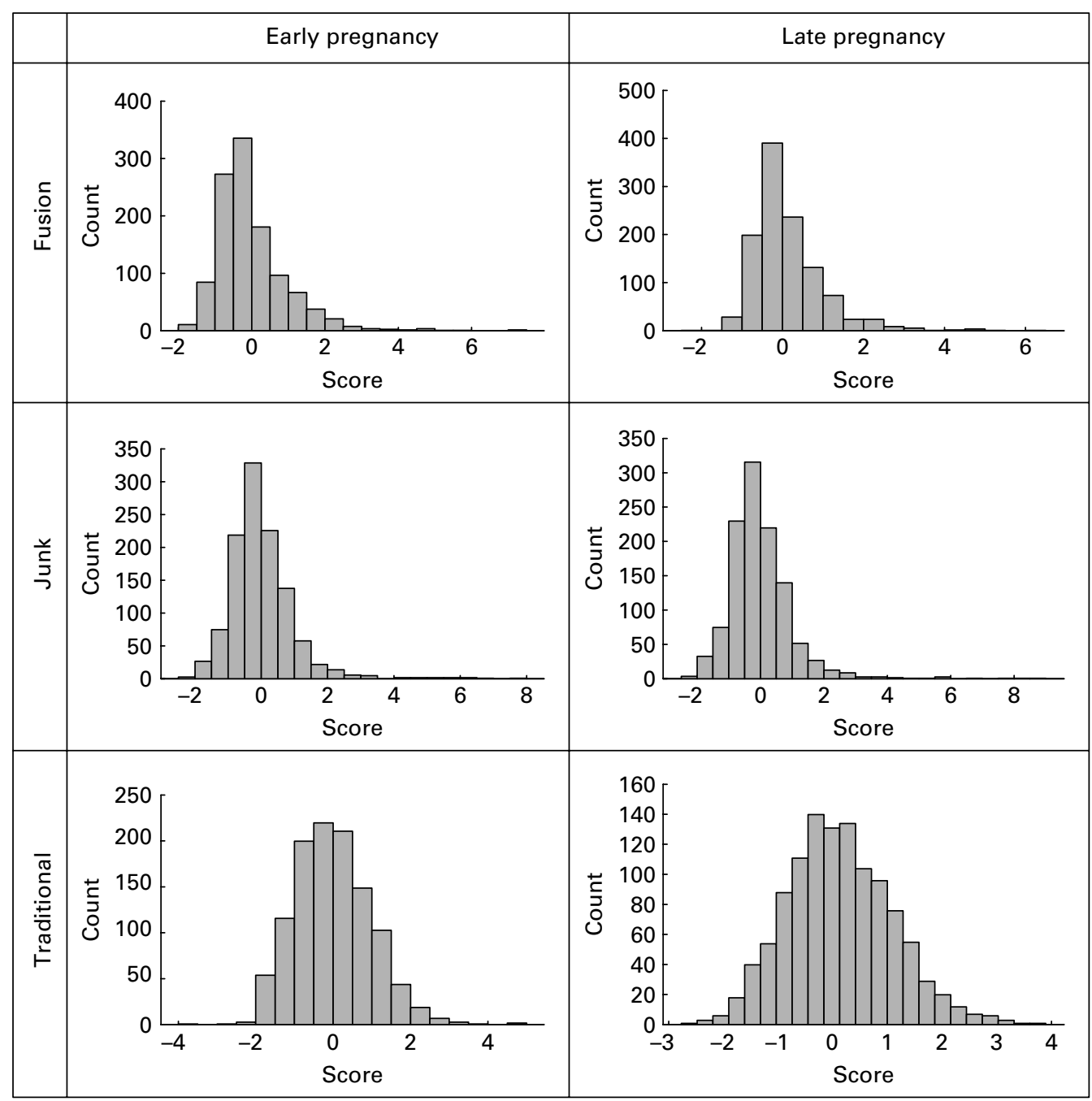

Fig. 1. Distribution of factor scores for diet in early and late pregnancy.

late pregnancy data. Analysis of non-standardised late pregnancy data revealed means of 0.13 (SD 0.88), 0.00 (SD 1.06) and 0.14 (SD 0.97). The correlation between the two time points for each dietary pattern was high. The correlation between the first and last months of pregnancy was 0.75 for the 'junk' diet, 0.74 for the traditional diet and 0.83 for the 'fusion' diet. In contrast, the correlation between the different dietary patterns, at the different time points, was poor. The relatively similar dietary score distributions in conjunction with the high correlations between early and late pregnancy suggest that there is little change in dietary pattern from the first to last month of pregnancy.

\section{Factors associated with dietary habits}

We analysed factors that were potentially related to mothers' choice of particular dietary patterns. We have only shown factors associated with early diet here, as the relationships with late diet were very similar.

For the junk diet, at the univariate level, women who were of lower or middle socio-economic status, were unmarried, smoked during pregnancy, were multiparous, had not attended antenatal classes and were heavier had an increased 'junk' diet score (Table 2). Furthermore, the score increased linearly with decreasing maternal age. In comparison to European women, the scores were higher for mothers of Maori and Pacific Island ethnicity and lower for Indian and Chinese women. When these variables were put into a multivariable model, only the relationship with ethnicity remained significant (Table 3).

For the traditional diet, women had higher scores if they were of high socio-economic status, left school after 16, were married or in a de facto relationship, did not smoke or use marijuana during pregnancy, were of average or taller than average height, of average weight and attended antenatal classes (Table 2). The score for this diet also increased linearly with maternal age and was higher among European women. Compared to European women, Maori, Pacific Island and Chinese women had statistically lower scores for this dietary pattern. In a multivariable analysis, those associations with marital status, maternal weight, maternal age and those of Maori and Chinese ethnicity remained statistically significant (Table 3).

For the 'fusion' diet, women of low or middle socio-economic status, of unmarried status and of average or shorter than normal height had higher scores. The score also increased with decreasing maternal age. Compared to European women, women of all other ethnicities had higher scores for this 


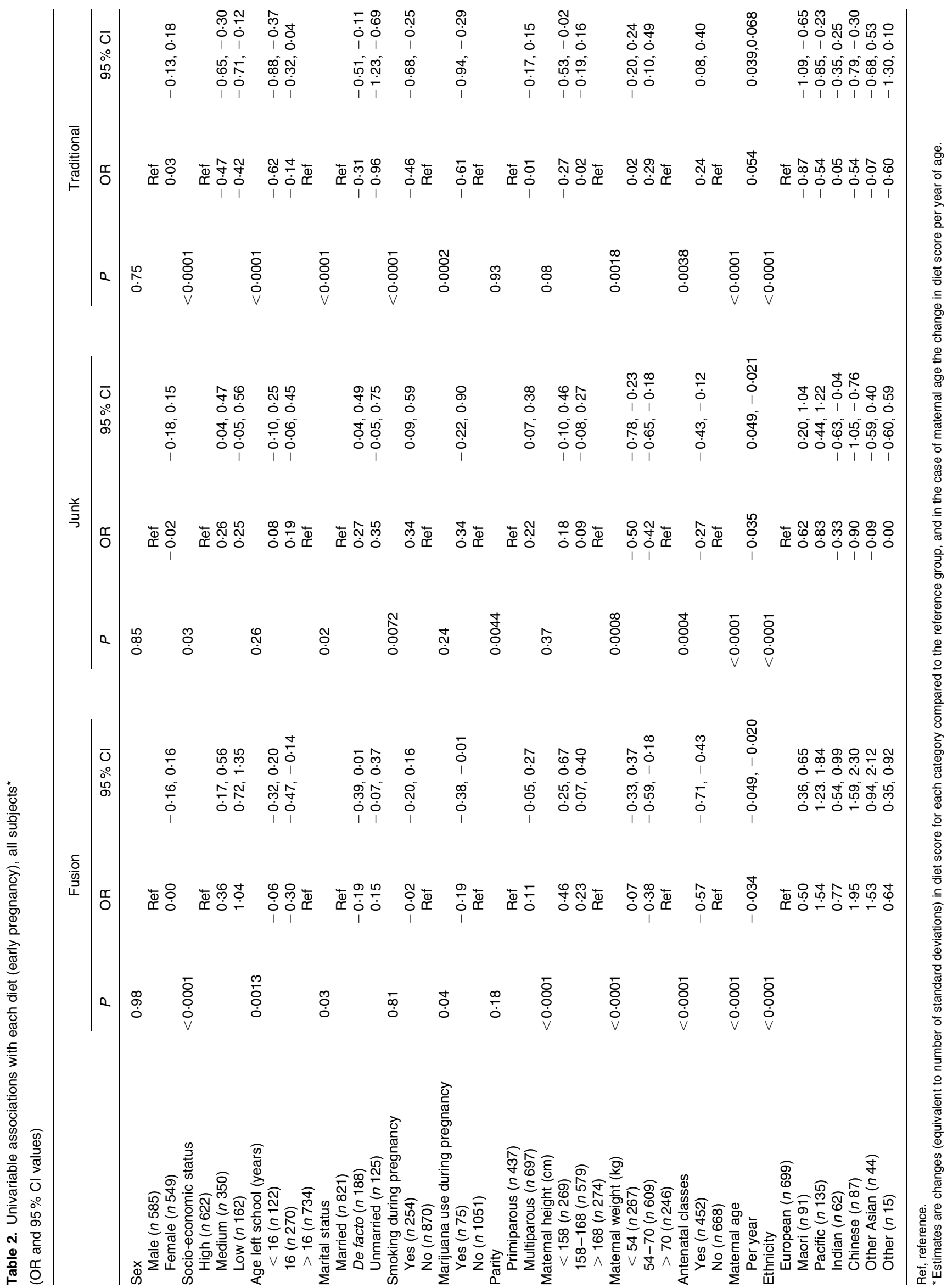


Table 3. Multivariable risk factors for each diet (early pregnancy) ${ }^{\star}$

(OR and $95 \% \mathrm{Cl}$ values)

\begin{tabular}{|c|c|c|c|c|c|c|c|c|c|}
\hline & \multicolumn{3}{|c|}{ Fusion } & \multicolumn{3}{|c|}{ Junk } & \multicolumn{3}{|c|}{ Traditional } \\
\hline & $P$ & OR & $95 \% \mathrm{Cl}$ & $P$ & OR & $95 \% \mathrm{Cl}$ & $P$ & OR & $95 \% \mathrm{Cl}$ \\
\hline Socio-economic status & 0.0048 & & & & & & & & \\
\hline High & & Ref & & & & & & & \\
\hline Medium & & 0.14 & $-0.02,0.31$ & & & & & & \\
\hline Low & & 0.39 & $0.11,0.68$ & & & & & & \\
\hline Marital status & & & & & & & 0.0026 & & \\
\hline Married & & & & & & & & Ref & \\
\hline De facto & & & & & & & & -0.19 & $-0.40,0.03$ \\
\hline Unmarried & & & & & & & & -0.63 & $-0.99,-0.27$ \\
\hline Maternal weight $(\mathrm{kg})$ & & & & & & & 0.02 & & \\
\hline$<54$ & & & & & & & & 0.01 & $-0.23,0.25$ \\
\hline $54-70$ & & & & & & & & 0.21 & $0.02,0.40$ \\
\hline$>70$ & & & & & & & & Ref & \\
\hline Maternal age & & & & & & & 0.0099 & & \\
\hline Per year & & & & & & & & 0.023 & $0.006,0.040$ \\
\hline Ethnicity & $<0.0001$ & & & $<0.0001$ & & & 0.0004 & & \\
\hline European & & Ref & & & Ref & & & Ref & \\
\hline Maori & & 0.48 & $0.29,0.67$ & & 0.25 & $-0.09,0.59$ & & -0.33 & $-0.60,-0.06$ \\
\hline Pacific & & 1.36 & $1.03,1.69$ & & 0.71 & $0.31,1.12$ & & -0.25 & $-0.59,0.09$ \\
\hline Indian & & 0.67 & $0.45,0.90$ & & -0.24 & $-0.56,0.07$ & & 0.10 & $-0.42,0.21$ \\
\hline Chinese & & 1.81 & $1 \cdot 45,2 \cdot 18$ & & -0.94 & $-1 \cdot 16,-0.72$ & & -0.62 & $-0.90,-0.35$ \\
\hline Other Asian & & 1.49 & $0.93,2.05$ & & -0.07 & $-0.57,0.42$ & & -0.13 & $-0.77,0.51$ \\
\hline Other & & 0.37 & $0.08,0.65$ & & -0.09 & $-0.76,0.58$ & & -0.56 & $-1 \cdot 20,0.07$ \\
\hline
\end{tabular}

Ref, reference.

* Estimates are changes (equivalent to number of standard deviations) in diet score for each category compared to the reference group, and in the case of maternal age the change in diet score per year of age. Only significant variables shown.

diet. In multivariable analysis, the associations with socioeconomic status and ethnicity remained significant.

\section{Associations between dietary patterns and birth weight of infant}

In logistic regression models of SGA, at a univariate level, traditional diet scores in early pregnancy showed a protective effect for an SGA infant $(\mathrm{OR}=0.79 ; 95 \%$ CI $0.70,0.89$; per standard deviation change in score), while in late pregnancy, the effect was similar $(\mathrm{OR}=0.83$; $95 \%$ CI 0.74 , 0.94; Table 4). When both time periods were put in a model together, the effect of diet in early pregnancy remained significantly associated with SGA and that of late pregnancy was no longer significant (Table 4). The junk and fusion diet scores showed no significant effect on SGA.

When the early diet factor scores were added to the main model that has been published previously ${ }^{(2)}$, the traditional diet score showed a reduced risk of SGA $(\mathrm{OR}=0.89 ; 95 \%$ CI $0.78,1.03$ ), the removal of non-significant variables from this model rendered this effect significant at the $5 \%$ level $(\mathrm{OR}=0.86 ; 95 \%$ CI $0.75,0.99)$. The final model included gestational age, infant sex, maternal smoking during pregnancy, maternal height and pre-pregnancy weight, primiparity, ethnicity and maternal hypertension. Other variables controlled for but removed from the final model as they were no longer significant were socio-economic status, age mother left school, marital status, marijuana use during pregnancy and attendance at antenatal classes. All variables remaining

Table 4. Univariable and multivariable associations between diet and small-for-gestational-age infants, all subjects (OR and $95 \% \mathrm{Cl}$ values)

\begin{tabular}{|c|c|c|c|c|c|c|}
\hline \multirow[b]{2}{*}{ Diet type } & \multicolumn{2}{|c|}{ Univariable model } & \multicolumn{2}{|c|}{ Multivariable model $^{*}$} & \multicolumn{2}{|c|}{ Final multivariable model $†$} \\
\hline & OR & $95 \% \mathrm{Cl}$ & OR & $95 \% \mathrm{Cl}$ & OR & $95 \% \mathrm{Cl}$ \\
\hline \multicolumn{7}{|c|}{ Diet in early pregnancy } \\
\hline Fusion & 1.07 & $0.95,1.21$ & $1 \cdot 15$ & $0.91,1.14$ & 1.02 & $0.85,1.21$ \\
\hline Junk & 0.97 & $0.86,1.09$ & 0.99 & $0.82,1 \cdot 18$ & 1.01 & $0.88,1.17$ \\
\hline Traditional & 0.79 & $0.70,0.89$ & 0.79 & $0.66,0.95$ & 0.86 & $0.75,0.99$ \\
\hline \multicolumn{7}{|c|}{ Diet in late pregnancy } \\
\hline Fusion & 1.05 & $0.92,1.20$ & 0.91 & $0.90,1.18$ & & \\
\hline Junk & 0.97 & $0.87,1.08$ & 0.99 & $0.83,1.17$ & & \\
\hline Traditional & 0.83 & $0.74,0.94$ & 1.01 & $0.84,1.23$ & & \\
\hline
\end{tabular}

* Multivariable model includes dietary scores for early and late pregnancy.

† Mulitvariable model controls for gestation, infant sex, maternal smoking in pregnancy, maternal pre-pregnancy height and weight, parity, ethnicity and maternal hypertension. 
in the model had similar significance levels and OR as in the original published model.

\section{Discussion}

We have shown that semi-quantitative FFQ used during pregnancy can be used to describe maternal dietary patterns. Additionally, having defined these dietary patterns, we were able to describe the relationship between pattern of diet that a mother is consuming and the effect of this diet in relation to giving birth to an SGA infant, namely that mothers who eat a 'traditional' diet during pregnancy were less likely to give birth to an SGA infant. Our analysis has also shown that a number of factors, particularly socio-demographic factors, are related to diet. We and others have previously shown that lower socio-economic status is related to birth weight and an increased risk of an SGA infant ${ }^{(2)}$. After controlling for these variables, there continued to be a lower risk of SGA, as the score for the 'traditional' dietary pattern increased.

Until very recently, there have been little data on dietary patterns of mothers during pregnancy. One older study in Mexican-American women described seven dietary factors ${ }^{(21)}$; similarly, a Finnish study also found seven dietary patterns ${ }^{(22)}$; however, they chose a relatively low factor loading score of $0 \cdot 2$, compared to $0 \cdot 3$ used by most other studies. Beyond the first two dietary patterns, the additional percentage of variance explained was relatively low. A recent publication from a large Danish study has described two dietary factors that they used to categorise women into three dietary patterns ${ }^{(23)}$. An analysis from the Avon Longitudinal Study of Pregnancy and Childhood in Britain has described five dietary factors $^{(24)}$, while data from a Southhampton study defined two dietary patterns termed 'prudent' and 'Western'(25).

A more in-depth study of diet that collected data six times from pre-conception to 6 months postnatally, in a group of eighty women, in Spain, described two dietary patterns, described as 'sweetened beverages and sugar' and 'vegetables and meat' ${ }^{\text {(26) }}$. It is notable that the factor loadings across time varied considerably using this methodology as shown by their coefficients of congruence, which were good between some time points but poor between others. In comparison, our use of the same dietary definition at both time points showed good correlation between the dietary scores in early and late pregnancy. This in conjunction with similar distributions of dietary scores in early and late pregnancies indicates a relative lack of change in diet during pregnancy for individuals.

The foods and dietary pattern described in our three dietary types are similar to that previously described. For consistency, we have used similar terminology to describe them. While the range of foods eaten across different countries and cultures varies and hence there will always be differences in the questionnaires used, the relative consistency of dietary factors produced in factor analyses is reassuring and confirms the usefulness of this type of analysis for semi-quantitative FFQ.

The study in Mexican-American mothers found that nutrient-dense and protein-rich foods were associated with an increase in infant birth weight, while a diet termed traditional that contained fats and oils, high-fat meats and sugar was associated with a decrease in birth weight ${ }^{(21)}$. The recent results of the Danish study by Knudsen et al. ${ }^{(23)}$ showed that those mothers who had the highest intakes of red and processed meats, potatoes and high dairy fat with low intakes of fruits and vegetables had the lowest birth weights. Additionally, when the present study defined three groups from their two dietary types, they found a decreased risk of having an SGA infant among the healthier diets ${ }^{(23)}$. The British study found the lowest birth weights associated with processed and vegetarian diets and the highest birth weights associated with health conscious diets ${ }^{(27)}$. In general, there is concordance across all studies with better outcomes in terms of birth weight with what are perceived as healthier diets.

Anecdotally, eating of junk food is associated with increased levels of obesity; however, there is no evidence to our knowledge on human subjects as to the reasons for this (apart from energy balance). Some animal studies do give us an insight into the possible mechanisms. In a study in which rats were fed four times their normal lard intake compared to control rats, the lard fed rats ate less but had the same energy intake and there was no effect on birth weight ${ }^{(28)}$. Another study that increased maternal food consumption in sheep by $50 \%$ increased birth weight, but suggested that the increased energy intake placed a different metabolic demand on the mother resulting in this energy being laid down as maternal fat ${ }^{(29)}$.

A study on rats fed a 'cafeteria' diet (which consisted of an ad libitum choice of palatable processed food with a high fat or high sugar content including muffins, jam doughnuts, biscuits, cheese, marshmallows, potato crisps and chocolate bars in addition to rat chow) showed no significant difference in birth weight of pups compared to controls; however, those pups whose mothers were continued on a cafeteria diet until weaning had increased perineal fat pads compared to controls and also to a group that reverted back to a normal diet after birth. Adiposity was avoided when pups were reverted back to the normal diet but muscle atrophy and fibre hypoplasia remained $^{(30,31)}$.

A further study with a similar design showed that those rats offered a cafeteria diet along with their normal diet ate $40 \%$ more food and had $56 \%$ more energy than controls, with only $20 \%$ of their total energy coming from their normal food. At the end of pregnancy, compared to controls, the junk diet rats were $13 \%$ heavier and there was no difference in litter size but a significant reduction in birth weight among the pups of approximately $4 \%^{(32)}$.

The parallels of this would suggest the human mothers who tend towards eating 'junk' diets not only eat less healthily but also eat more of this unhealthy food. The outcome in terms of early childhood is likely to depend on whether an infant is breastfed, a factor that differs significantly in prevalence across Western countries. The longer-term outcomes would appear more perilous, however, as one would expect the diet of the child to follow that of their parents as they become older.

There are limitations to any study investigating nutritional intake. We collected the information on early and late pregnancy dietary intakes retrospectively shortly after the birth of the child. Recall bias could be an issue, particularly for the early pregnancy data; we did, however, give cues to the mother about when this timeframe was, to help recall. We have also carried out a test-retest study using these questionnaires, with the mother filling out the questionnaires in early pregnancy and then again after the birth of the child. 
This found some regression towards the mean for intakes of foods ${ }^{(18)}$. This could explain some of the similarities in dietary patterns and weightings between the time points. This would suggest less variation in the retrospectively collected data than is present at the time of intake, hence making differences found to be potentially conservative. Collecting dietary intake via FFQ has its limitations but is the primary and practical way of collecting nutritional data in large epidemiological studies. The use of a FFQ in the present study to determine dietary habits enables a longer-term view of dietary intakes as opposed to using a 24-h dietary recall or food diary method. However, FFQ cannot represent the total diet and there are assumptions made about ingredients used for composite dishes. This FFQ has previously been validated in studies in younger children, which described dietary intakes and $\mathrm{Fe}$ status.

The methodology of using principal components analysis is becoming commonly used to reduce a large number of foods collected using FFQ to a smaller set of variables. It is ideal for analysing dietary data of the type collected here, and the results across similar populations appear to be relatively similar. There are of course weaknesses to using this method; the dietary patterns are not chosen a priori but are driven by the data and hence no two studies will identify identical diets, making direct comparison between studies impossible. Also, there are no strict cut-offs for the additional percentage of variance explained to decide on how many factors should be chosen or on the cut-off for loading scores to determine which foods should be considered important in each dietary pattern. The results obtained from these sorts of analyses to date and the sense of relationships of dietary patterns to socio-demographic variables and to outcomes such as birth weight enforce the appropriateness of using this type of analysis for data of this nature.

These results, if confirmed, have major implications for antenatal care and pregnancy planning. We have shown that although the effects of a 'traditional' diet were significant univariately for both early and late pregnancies, the major effect was that associated with early pregnancy. Although emphasis is placed on early and pre-pregnancy, for example, ensuring adequate intakes of folate to protect against neural tube defects, the present results add to the reasons for careful planning in early and pre-pregnancy. From a public health perspective, this will be difficult, as $45 \%$ of the pregnancies in the present study were unplanned.

The response rate among the European sample was high, and we can be confident that the results for this group are representative of this group of women. A limitation of the study, however, was the lower response rate seen among other ethnic groups. Previous analysis has shown the non-respondents are also more likely to be in the lower socio-economic groups. There needs to be caution in interpreting the results in other ethnic groups.

In conclusion, maternal diet particularly in early pregnancy is important for the development of the fetus. Sociodemographic factors tend to be significantly related to dietary pattern, suggesting that extra resources may be necessary for disadvantaged mothers so that they can purchase more healthy foods, which tend to be more expensive than high-energy foods. Eating well during pregnancy is essential for the future health of the unborn child.

\section{Acknowledgements}

The present study was funded by the Health Research Council of New Zealand (HRC) and the Foundation for the Newborn. Prof. Mitchell and Dr Thompson are currently funded by the Child Health Research Foundation. We would like to thank J. Birkbeck and Auckland Dietetic Services for advice on nutritional aspects of the study, the obstetric, midwifery and ward staff at the three hospitals and the mothers who took part. We would also like to acknowledge the effort put into the study by all the interviewers. J. M. D. T. was involved with the study design, collection of the data, analysis and wrote the initial manuscript. C. W. helped with the analysis and interpretation of the data and writing of the manuscript. D. M. O. B., E. R. and C. J. W. were involved with the design of the study, helped to interpret the analysis and contributed to the writing of the paper. E. A. M. is the principal investigator and was involved with the design of the study, interpretation of the results and writing of the manuscript. The authors confirm that they have no conflicts of interest.

\section{References}

1. Kramer MS (1987) Determinants of low birth weight: methodological assessment and meta-analysis. Bull World Health Organ 65, 663-737.

2. Thompson JM, Clark PM, Robinson E, et al. (2001) Risk factors for small-for-gestational-age babies: the Auckland Birthweight Collaborative study. J Paediatr Child Health 37, 369-375.

3. Painter RC, Roseboom TJ \& Bleker OP (2005) Prenatal exposure to the Dutch famine and disease in later life: an overview. Reprod Toxicol 20, 345-352.

4. Roseboom T, de Rooij S \& Painter R (2006) The Dutch famine and its long-term consequences for adult health. Early Hum Dev 82, 485-491.

5. Roseboom TJ, van der Meulen JH, Ravelli AC, et al. (2001) Effects of prenatal exposure to the Dutch famine on adult disease in later life: an overview. Twin Res 4, 293-298.

6. Stein AD, Zybert PA, van de Bor M, et al. (2004) Intrauterine famine exposure and body proportions at birth: the Dutch Hunger Winter. Int J Epidemiol 33, 831-836.

7. Smith C (1947) The effects of wartime starvation in Holland on pregnancy and its product. Am J Obstet Gynecol 53, 599-608.

8. Harding JE (2001) The nutritional basis of the fetal origins of adult disease. Int $J$ Epidemiol 30, 15-23.

9. Harding JE (2003) Nutrition and growth before birth. Asia Pac $J$ Clin Nutr 12, S28.

10. Heasman L, Clarke L, Stephenson TJ, et al. (1999) The influence of maternal nutrient restriction in early to mid-pregnancy on placental and fetal development in sheep. Proc Nutr Soc 58, 283-288.

11. Tardif S, Power M, Layne D, et al. (2004) Energy restriction initiated at different gestational ages has varying effects on maternal weight gain and pregnancy outcome in common marmoset monkeys (Callithrix jacchus). Br J Nutr 92, 841-849.

12. Bower C, Stanley FJ \& Nicol DJ (1993) Maternal folate status and the risk for neural tube defects. The role of dietary folate. Ann N Y Acad Sci 678, 146-155.

13. Murphy M, Whiteman D, Stone D, et al. (2000) Dietary folate and the prevalence of neural tube defects in the British Isles: the past two decades. BJOG 107, 885-889.

14. Shaw GM, Schaffer D, Velie EM, et al. (1995) Periconceptional vitamin use, dietary folate, and the occurrence of neural tube defects. Epidemiology 6, 219-226. 
15. Steegers-Theunissen RP (1995) Folate metabolism and neural tube defects: a review. Eur J Obstet Gynecol Reprod Biol 61, 39-48.

16. Barker DJ, Osmond C, Forsen TJ, et al. (2005) Trajectories of growth among children who have coronary events as adults. $N$ Engl J Med 353, 1802-1809.

17. Lucas A, Fewtrell MS \& Cole TJ (1999) Fetal origins of adult disease - the hypothesis revisited. BMJ 319, 245-249.

18. Mitchell EA, Robinson E, Clark PM, et al. (2004) Maternal nutritional risk factors for small for gestational age babies in a developed country: a case-control study. Arch Dis Child Fetal Neonatal Ed 89, F431-F435.

19. Thompson JM, Mitchell EA \& Borman B (1994) Sex specific birthweight percentiles by gestational age for New Zealand. $N$ Z Med J 107, 1-3.

20. Life iNZ (1991) Commission Report Prepared by University of Otago. Hillary Commission for Recreation and Sport. Wellington.

21. Wolff CB \& Wolff HK (1995) Maternal eating patterns and birth weight of Mexican American infants. Nutr Health 10, 121-134.

22. Arkkola T, Uusitalo U, Kronberg-Kippila C, et al. (2008) Seven distinct dietary patterns identified among pregnant Finnish women - associations with nutrient intake and sociodemographic factors. Public Health Nutr 11, 176-182.

23. Knudsen VK, Orozova-Bekkevold IM, Mikkelsen TB, et al. (2007) Major dietary patterns in pregnancy and fetal growth. Eur J Clin Nutr 62, 463-470.

24. Northstone K, Emmett P \& Rogers I (2007) Dietary patterns in pregnancy and associations with socio-demographic and lifestyle factors. Eur J Clin Nutr 62, 471-479.
25. Crozier SR, Inskip HM, Godfrey KM, et al. (2008) Dietary patterns in pregnant women: a comparison of food-frequency questionnaires and $4 \mathrm{~d}$ prospective diaries. $\mathrm{Br} J$ Nutr 99, 869-875.

26. Cuco G, Fernandez-Ballart J, Sala J, et al. (2006) Dietary patterns and associated lifestyles in preconception, pregnancy and postpartum. Eur J Clin Nutr 60, 364-371.

27. Northstone K, Ness AR, Emmett PM, et al. (2008) Adjusting for energy intake in dietary pattern investigations using principal components analysis. Eur J Clin Nutr 62, 931-938.

28. Khan IY, Taylor PD, Dekou V, et al. (2003) Genderlinked hypertension in offspring of lard-fed pregnant rats. Hypertension 41, 168-175.

29. Budge H, Gnanalingham MG, Gardner DS, et al. (2005) Maternal nutritional programming of fetal adipose tissue development: long-term consequences for later obesity. Birth Defects Res C Embryo Today 75, 193-199.

30. Bayol SA, Simbi BH \& Stickland NC (2005) A maternal cafeteria diet during gestation and lactation promotes adiposity and impairs skeletal muscle development and metabolism in rat offspring at weaning. $J$ Physiol 567, Pt 3, 951-961.

31. Holemans K, Caluwaerts S, Poston L, et al. (2004) Diet-induced obesity in the rat: a model for gestational diabetes mellitus. Am J Obstet Gynecol 190, 858-865.

32. Bayol SA, Farrington SJ \& Stickland NC (2007) A maternal 'junk food' diet in pregnancy and lactation promotes an exacerbated taste for 'junk food' and a greater propensity for obesity in rat offspring. Br J Nutr 98, 843-851. 